\title{
Green Banking, Corporate Governance and Performance of Selected SAARC Countries
}

\author{
Umara Ikram a , Shahzad Akhtar ${ }^{\text {b }}$ \\ ${ }^{a}$ PhD, Scholar Institute of Management Sciences, Bahauddin Zakariya University Multan, Pakistan \\ Email: umarasoe@gmail.com \\ ${ }^{\mathrm{b}}$ Assistant professor Institute of Management Sciences, Bahauddin Zakariya University Multan, Pakistan
}

\begin{tabular}{l} 
ARTICLE DETAILS \\
\hline History: \\
Accepted 15 December 2021 \\
Available Online December 2021
\end{tabular}

\section{Keywords:}

Green Banking, Corporate

Governance, Firm Performance

\section{JEL Classification:}

G24,

DOI: $10.47067 /$ reads.v7i4.415

\begin{abstract}
This study is designed to estimate impact of green banking disclosure, corporate governance mechanism on performance of listed banks in selected SAARC countries including Pakistan, India, Bangladesh, Sri Lanka and Nepal. With the help of STATA 14.2 this study used PCA (Principal Component Analysis) in addition to content analysis to create green banking disclosure index .For this purpose, central bank's green banking guidelines are summarized into7 categories and 38 items. Dynamic panel data set (2010-2019) is analyzed by applying system GMM step-one method. The relationships among board independence, board size, female director, institutional ownership, green banking and Tobin's $Q$ (market value) as performance measure is tested. Institutional ownership and board independence has significant negative impact on market value, green banking does not have any significant impact on market value. On average disclosure practices are different in different categories. Effectiveness of central bank guidelines can be identified at regional level. Results are suggestive that corporate governance mechanism restructuring is needed to increase market value of banks in SAARC countries. To the best of author's knowledge, this is the very first study which methodologically contributes in the field of green banking disclosure as application of PCA and System GMM step-one. Contextually, one of the most affected area facing higher climate change risk as SAARC region of the world is discussed. Theoretically, study contributes in the theory of change, financial intermediation and agency theory.
\end{abstract}

(C) 2021 The authors. Published by SPCRD Global Publishing. This is an open access article under the Creative Commons Attribution-

NonCommercial 4.0

Corresponding author's email address: umarasoe@gmail.com

\section{Introduction}

According to UN agenda 2030 for sustainable development, it is reported that GHG emission levels are increasing. The latest IPCC report (IPCC 2018) declares that human activities are causing global warming which is likely to accelerate further by reaching $1.5{ }^{\circ} \mathrm{C}$. Global climate risk index 2018 
Review of Economics and Development Studies, Vol. 7 (4) 2021, 543 - 559

provides list of countries with long term and short term climate risk. Most affected countries belonging to SAARC regions are Sri Lanka, India, Bangladesh and Pakistan. To reduce negative impacts, central banks, supervisors and policy makers started undertaking various green banking initiatives. Although practices are relatively different between developing and developed countries. At the global level, a network called the Network for Greening the Financial System (NGFS) has also been established by the central banks and regulators to address climate risks. Bangladesh has issued green banking guidelines in year 2011, 2012, 2013. India almost after 2012 all banks are directed to follow green coin rating guidelines. State Bank of Pakistan has announced green banking guidelines in 2017.Corporate governance refers to the rules, regulations procedures and structures by which the affairs of business and institutions are managed and directed, to enhance shareholder's value through improving corporate accountability and performance while considering the interest of other shareholders (Jenkins on\& Mayer, 1992). Alexander (2016) says that there is no universal definition of green banking and it varies across the countries. Park and Kim (2020) declares that green banking term is more similar to ethical banking, social or responsible banking or sustainable banking. Presently, there is need to have a unique, comprehensive measure by which different initiatives regarding green banking practices can be examined in different countries. In addition, there is immense potential to explore unobserved contribution of green banking practices on bank performance. Mostly, green banking literature consists of primary, cross sectional, descriptive and exploratory studies on the topics like, Green banking practices in India (Sudhalakshmi\& Chinnadorai,2014).Factors determining adoption of green banking among commercial banks in Malaysia (Arumugan and Chirute,2018)Measuring green banking practices in Sri Lanka (Shumya and Arulrojah,2016). Therefore, wide research gap is identified by research in the following areas, measuring green banking disclosure practices. Linking corporate governance mechanism and green banking with firm performance. The purpose of this research is to create green banking disclosure index by combining all central bank guidelines from selected SAARC countries. In addition, this study aims to identify the influencing effects of green banking disclosure index with corporate governance mechanism such as 1. Board size, 2.Board independence, 3. Female in board and institutional ownership on market value of banks belonging to selected SAARC countries.

\section{Related Literature Review}

Islam et al (2017) examine the impact of regulatory guidance and other factors on the green banking disclosure practices of Bangladeshi commercial banks in the period from 2007 to 2014. They find that, the issuance of green banking regulatory guidance by the Central Bank of Bangladesh in 2011 positively influences the level of green banking disclosure. They also report that green banking disclosure practices in the banking sector have converged over the time and have become a routine process. In addition, by following OLS model they find that corporate governance mechanisms (e.g., board size and institutional ownership) positively affect the level of green banking disclosure. However, this study finds no relationship between the presence of independent directors on the board and green banking disclosure. Dewi and Dewi (2017) provide empirical evidence about influencing role of green banking implementation on the relationship between corporate social responsibility and going concern value of banking companies in stock exchange of Indonesia. By applying moderated regression analysis (MRA) quantitative data (2013-2015) is analyzed and findings indicate implementation of green banking strengthens the relationship between corporate social responsibility and going concern value of banking companies in Indonesia. Wu et al (2019) establish a dynamic panel model for 12 Chineselisted commercial banks and seven international commercial banks. The impact of green credit on the profitability of commercial banks and the difference between China and other countries is examined by using the generalized method of moments. The research shows that the Equatorial Principles projectfinancing ratio of international banks positively affects bank profitability, while the ratio of green credit for Chinese commercial banks is inversely related to their profitability. 
Review of Economics and Development Studies, Vol. 7 (4) 2021, 543 - 559

Karim et al (2020) examines the effects of green banking practices on the financial performance of banks listed in the DSE of Bangladesh covering the period from 2011 to 2020. By using the panel data set, taking financial variables like return on asset, return on equity, and market value to proxy the banks' performance, and employing green banking practice variables like green cost and volume of the risk management committee, study concludes that there is a positive relationship between green banking practices and financial performance. Monem et al (2020) provide useful insight to examine whether bank's green performance can effect financial performance and whether this relation is moderated by bank's political connection. From Bangladesh, Sample of 172 firm-year observations from 2008-2014 by applying difference-in-difference (DiD),propensity score matching (PMS)analysis and Heckman's two stage analysis suggest that green banking performance is positively associated with banks financial performance. Robust findings also highlights political connections of banks negatively affects this relationship. Karyani and Obrien (2020) examine the effect of green banking practice on bank performance with foreign and public ownership as moderating variables of 14 Indonesian banks with 98 bank year observations between 2012 and 2018. By applying OLS (ordinary least square) model, this study provides useful insights that green banking practices have a negative impact on bank profitability but a positive impact on firm value. Negative effect of green banking practice on profitability is strengthen by public ownership. Positive impact of green banking practice on bank value is weakened by foreign ownership. Quazi et al (2021) builds on key insight whether combining green banking disclosure with contextual factor such as non-performing loans provides additional understanding about green banking disclosure and firm value. By analyzing seven years data of listed banks in Bangladesh (2008-2014) using multiple regression, they conclude that green banking disclosure gas positive effect on overall firm value. This positive effect is negatively moderated by banks non-performing loan. Gerged and Agwili (2019) identify in what way corporate governance affect firm profitability and firm value. A sample from (2012-2016) of 300 listed non-financial and financial companies from Saudi Arabia is analyzed by fixed effect panel data regression and GMM method. Results are suggestive that better governed firms tend not to improve accounting value but market value.

\section{Data, Variable and Methodology}

Keeping in consideration data protocols, the data covers the listed banks in respective stock exchanges including Bangladesh, Pakistan, Sri Lanka, India and Nepal from 2010-2019 according to their annual reports. Banks with incomplete data were excluded from sample (Rehman,2016).Data regarding stock prices have been calculated either through stock price history information available stock exchanges, annual report year averages or from Investing.com to calculate market value of firms. Final sample includes 32 banks with 320 total 10 year observation. Sample comprises of 9 banks from India, 9 banks from Bangladesh, 5 Banks from Sri Lanka, 5 banks from Pakistan and 4 Banks from Nepal. The study analyses data on green banking disclosure practices by manually coding information on 38 items as 1 if information is present and o otherwise from annual reports available on website. These 38 items comprises of following categories, 1.Environment risk management 2.Green banking facilitation 3.Guidelines on own impact reduction. 4. Management related guidelines 5.Organization related guidelines 6.Green business facilitation 7. Specific guidelines. (SBP 2017). Then green banking disclosure index is developed by Principle Component Analysis technique (Al-Homaidi et al, 2021). The method of Principal Component Analysis is considered extremely reliable and accurate technique for empirical investigation of non-financial disclosure (Popa et al, 2021).

\section{Definition of Variables}

Over variables of interest regarding corporate governance are board size (BRDSIZE) which is measured as total number of directors in board, board independence (BRDIND) measured as number of 
Review of Economics and Development Studies, Vol. 7 (4) 2021, 543 - 559

independent directors in board, institutional ownership (INSTOWN) is measured as percentage of ownership holdings by institutional investors. Female director (FD) is measured as total number of females .A number of control variables are also included for controlling firm specific characteristics. Firm size (FSIZE) is measured as the natural logarithm of the firm's total assets. Firm age (FAGE) is measured as total number of years from inauguration. Leverage (LEV) is measured as the ratio of total debt to total assets whereas profitability (ROA) is measured as the ratio of net income over total assets. (Islam et al, 2017).Green banking disclosure practices are measured by constructing green banking disclosure Index (GBDI). List of 38 items with key words is provided in appendix 1.

The definition of these variables along with variable type and source is given below in table.

Table: 1 Definitions of variables, types and sources.

\begin{tabular}{|l|l|l|l|}
\hline Variables & Measurement & Variable Type & Source \\
\hline BRDSIZE & Total number of directors in board. & Independent & Annual report \\
\hline BRDIND & $\begin{array}{l}\text { Number of independent directors in } \\
\text { board. }\end{array}$ & Independent & Annual report \\
\hline INSTOWN & $\begin{array}{l}\text { Percentage of ownership holdings by } \\
\text { institutional investors }\end{array}$ & Independent & Annual report \\
\hline FD & Total number of females in board. & Independent & Annual report \\
\hline FSIZE & $\begin{array}{l}\text { The natural logarithm of the firm's } \\
\text { total assets. }\end{array}$ & Control & Annual report \\
\hline FAGE & $\begin{array}{l}\text { Total number of years from } \\
\text { inauguration }\end{array}$ & Control & Annual report \\
\hline LEV & $\begin{array}{l}\text { The ratio of total debt to total assets } \\
\text { assets }\end{array}$ & Control & Annual report \\
\hline ROA & Green banking disclosure Index & Independent & Annual report \\
\hline GBDI & $\begin{array}{l}\text { Tobin's Q }=\text { Total Asset+ Market value } \\
\text { of equity-Book value of Equity/Total } \\
\text { Asset }\end{array}$ & $\begin{array}{l}\text { Dependent } \\
\text { Variable }\end{array}$ & Annual Report \\
\hline (Tobin's Q) & \multicolumn{2}{|l|l|}{ Control } & \\
\hline
\end{tabular}

\section{Econometric Model and Methodology}

Firm performance is measured as market value of firm by Tobin's Q.(Batsakis et al, 2018).To examine the impact of corporate governance mechanism and green banking disclosure index on market value of banks, following econometric model is developed by taking in consideration all variables mentioned in table above.



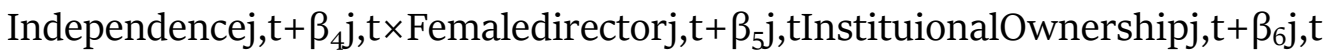

Green Banking Index $\mathrm{j}, \mathrm{t}+\beta_{\mathrm{j}} \mathrm{j}, \mathrm{t} \times$ Controlsj, $\mathrm{t}+€ \mathrm{j}, \mathrm{t}$

(1)Firm Valuej, $t=$ Tobin's $Q$ of firm $j$ at time $t$.

(2)Firm Valuej,t-1= Tobin's Q of firm $\mathrm{j}$ at time $\mathrm{t}-1$.

(3)Board Sizej, $t=$ Board size of firm $\mathrm{j}$ at time $\mathrm{t}$.

(4)Board Independence $\mathrm{j}, \mathrm{t}=$ Total independent director of firm $\mathrm{j}$ at time $\mathrm{t}$.

(5)Female director $\mathrm{j}, \mathrm{t}=$ Female directors of firm $\mathrm{j}$ at time $\mathrm{t}$.

(6)Institutional Ownership $\mathrm{j}, \mathrm{t}=\%$ of institutional ownership of firm $\mathrm{j}$ at time $\mathrm{t}$. 
(7)Green Banking Index $\mathrm{j}, \mathrm{t}=$ Green Banking index of firm $\mathrm{j}$ at time $\mathrm{t}$.

(8)Controls $\mathrm{j}, \mathrm{t}=$ Control variables of firm $\mathrm{j}$ at time $\mathrm{t}$.

According to Jatmiko et al (2020) corporate governance variables including board size, board independence and female in board are dynamic in nature. Firm value is measured as Tobin's Q which is lag dependent variable. Most of previous work (Bitar et al, 2017) in banking have been practicing pooled OLD estimation However, according to Baltagi (2008), pooled analysis using random or fixed effects are biased even if the error term is not serially correlated. That's why, this work used system GMM to test the dynamic relationship between Firm value, corporate governance characteristics and green banking disclosure in the presences of control variables which are bank specific. According to Judson and Owen (1999) for dynamic panel data estimation, system GMM one step is highly recommended when Time period is less than or equal to 10 as in the case of current study. Alqahtani and Mayes (2018) in comparison to other panel methods, system GMM have advantages such as dynamic modeling treats autocorrelation, endogeneity, and unobserved heterogeneity. System GMM (Arellano-Bond estimation) is available in two versions, one step and two step. The asymptotic standard error of estimation of one step is more reliable and unbiased to draw inferences but at the same time in the case of heteroscedasticity, it cannot produce Sargan statistics. In this case one can rely Wald-Chi statistics to check over-identification restriction and overall significance of the model. (Pandy and Sahu, 2021)

\section{Findings and Discussion on Results}

Table 2: Summary of methodologies used in green banking literature

\begin{tabular}{|c|c|c|c|}
\hline Author(year) & Sample & Determinants & Methods \\
\hline Islam et al (2017) & $\begin{array}{l}\text { 30 Bangladesh Bank } \\
2007-2014\end{array}$ & $\begin{array}{lr}\text { Board size, } & \text { Board } \\
\text { independence, } & \text { Female } \\
\text { director ,Institutional } \\
\text { ownership, } & \text { Growth } \\
\text { opportunities, } & \text { Year } \\
\text { dummy, Firm } & \text { size }, \text { Lev, } \\
\text { ROA Firm age, }\end{array}$ & OLS regression. \\
\hline Dewi \& Dewi(2017) & $\begin{array}{l}\text { 10 Banks Indonesia } \\
(2013-2015)\end{array}$ & $\begin{array}{l}\text { CSR disclosure, Green } \\
\text { banking regulations, } \\
\text { Going concern value. }\end{array}$ & $\begin{array}{l}\text { Moderated } \\
\text { Regression } \\
\text { Analysis } \\
\text { (MRA) }\end{array}$ \\
\hline Karim et al (2020) & $\begin{array}{ll}10 & \text { listed } \\
\text { commercial banks } & \text { bank } \\
\text { china }(2011-2020)\end{array}$ & $\begin{array}{l}\text { ROA, ROE, Green credit } \\
\text { ratio as cost, Volume of } \\
\text { risk management } \\
\text { committee. }\end{array}$ & $\begin{array}{l}\text { Panel Data } \\
\text { Analysis }\end{array}$ \\
\hline Wu et al (2019) & $\begin{array}{l}19 \text { Chinese listed } \\
\text { bank } \\
(2008-2015)\end{array}$ & $\begin{array}{l}\text { Green credit ratio, ROA, } \\
\text { ROE, NPL, Capital } \\
\text { adequacy ratio. }\end{array}$ & $\begin{array}{l}\text { GMM, Dynamic } \\
\text { panel } \\
\text { analysis. }\end{array}$ \\
\hline Monem et al (2020) & $\begin{array}{l}172 \text { firm years } \\
\text { observations } \\
(2008-2014)\end{array}$ & $\begin{array}{l}\text { Green credit ratio, ROA, } \\
\text { Political connections. }\end{array}$ & $\begin{array}{l}\text { Difference in } \\
\text { Difference } \\
\text { (DiD), } \\
\text { Propensity } \\
\end{array}$ \\
\hline
\end{tabular}


Review of Economics and Development Studies, Vol. 7 (4) 2021, 543 - 559

\begin{tabular}{|c|c|c|c|}
\hline & & & $\begin{array}{l}\text { scoring method, } \\
\text { Heckman's two } \\
\text { stage analysis. }\end{array}$ \\
\hline Karyani \&Obrien (2020) & $\begin{array}{l}\text { Indonesian } \quad \text { Bank } \\
(2012-2018)\end{array}$ & $\begin{array}{l}\text { ROA, Green banking } \\
\text { practices, Foreign Public } \\
\text { Ownership. }\end{array}$ & OLS regression. \\
\hline Quazi et al (2021) & $\begin{array}{lc}\text { Listed } & \text { banks } \\
\text { Bangladesh } & (2008- \\
2014) & \end{array}$ & $\begin{array}{l}\text { Green banking disclosure, } \\
\text { non-performing loan, } \\
\text { Tobin's Q }\end{array}$ & $\begin{array}{l}\text { Multiple } \\
\text { Regression } \\
\text { Analysis. }\end{array}$ \\
\hline Gerged and Agwili (2019) & $(2012-2016)$ & \begin{tabular}{lr} 
Corporate & \multicolumn{2}{r}{ governance } \\
mechanism, & Market \\
value, ROA, ROE, Tobin's \\
Q, Board Size, \\
independence, \\
meeting,
\end{tabular} & $\begin{array}{l}\text { Fixed Effect } \\
\text { Panel data } \\
\text { regression. } \\
\text { GMM model. }\end{array}$ \\
\hline Gosh et al (2021) & $\begin{array}{l}30 \text { Banks } \\
(2011-2017)\end{array}$ & $\begin{array}{l}\text { Board Independence, } \\
\text { Board meetings, Board } \\
\text { diversity, Tobin's Q, ROA, } \\
\text { Audit committee size, } \\
\text { Non- executive directors. }\end{array}$ & $\begin{array}{l}\text { Pooled } \quad \text { OLS } \\
\text { Method. }\end{array}$ \\
\hline
\end{tabular}

Table: 3 Descriptive Data Statistics

\begin{tabular}{|l|l|l|l|l|l|}
\hline Variable & Obs & Mean & Std. Dev. & Min & Max \\
\hline Board Size & 320 & 11.5812 & 3.7416 & 5 & 22 \\
\hline Board Ind & 320 & 2.0031 & 2.1327 & 0 & 8 \\
\hline $\begin{array}{l}\text { Female } \\
\text { Director }\end{array}$ & 320 & 0.8937 & 0.9958 & 0 & 4 \\
\hline IO & 320 & 26.1703 & 23.4078 & 0 & 98.63 \\
\hline Firm Age & 320 & 42 & 32.3606 & 11 & 113 \\
\hline LEV & 320 & 77.6310 & 11.8855 & 16.64 & 92.03 \\
\hline ROA & 320 & 1.2440 & 1.7385 & -7.21 & 7.31 \\
\hline Firm Size & 320 & 26.7433 & 1.4741 & 23.0233 & 30.0802 \\
\hline Tobin's Q & 320 & 111.5365 & 43.8682 & 18.6244 & 636.5374 \\
\hline GB & 320 & 1.16 & 1.4142 & -0.3547 & 5.6200 \\
\hline
\end{tabular}

According to the table above mentioned maximum size of board is 22 members and minimum is 5. Board independence varies from o -8. At max there are 4 females in board. Institutional ownership varies from o to $98 \%$ which is very high. Firm value varies from $18.62 \%$ to $636.53 \%$.Green banking disclosure shows very low value -.354 to very high level of disclosure that is 5.62 among the selected SAARC countries. 
Review of Economics and Development Studies, Vol. 7 (4) 2021, 543 - 559

Table: 4 Content Analysis Results of Green Banking Disclosure on 38 Items.

\begin{tabular}{|c|c|c|c|c|c|}
\hline Variable & Obs. & Mean & Std. De & Min & Max \\
\hline GB1 & 320 & .5468 & .4985 & $\mathrm{O}$ & 1 \\
\hline GB2 & 320 & .4687 & .4998 & $\mathrm{O}$ & 1 \\
\hline GB3 & 320 & .5156 & .5005 & o & 1 \\
\hline GB4 & 320 & .3968 & .4900 & $\mathrm{O}$ & 1 \\
\hline GB5 & 320 & .2656 & .4423 & $\mathrm{O}$ & 1 \\
\hline GB6 & 320 & .4375 & .4968 & o & 1 \\
\hline GB7 & 320 & .0812 & .2736 & o & 1 \\
\hline GB8 & 320 & .3468 & .4767 & $\mathrm{O}$ & 1 \\
\hline GB9 & 320 & .4656 & .4995 & o & 1 \\
\hline GB10 & 320 & .3218 & .4679 & o & 1 \\
\hline GB11 & 320 & .4687 & .4998 & $\mathrm{O}$ & 1 \\
\hline GB12 & 320 & .5468 & .4985 & $\mathrm{O}$ & 1 \\
\hline GB13 & 320 & .4687 & .4998 & $\mathrm{O}$ & 1 \\
\hline GB14 & 320 & .2968 & .4575 & $\mathrm{O}$ & 1 \\
\hline GB15 & 320 & .0218 & .1465 & o & 1 \\
\hline GB16 & 320 & .4156 & .4936 & $\mathrm{O}$ & 1 \\
\hline GB17 & 320 & .0218 & .1465 & o & 1 \\
\hline GB18 & 320 & .0375 & .1902 & $\mathrm{O}$ & 1 \\
\hline
\end{tabular}


Review of Economics and Development Studies, Vol. 7 (4) 2021, 543 - 559

\begin{tabular}{|c|c|c|c|c|c|}
\hline GB19 & 320 & .4937 & .5007 & 0 & 1 \\
\hline GB2O & 320 & .0093 & .0965 & $\mathrm{O}$ & 1 \\
\hline GB21 & 320 & .9250 & .2638 & $\mathrm{O}$ & 1 \\
\hline GB22 & 320 & .4593 & .4991 & o & 1 \\
\hline GB23 & 320 & .6250 & .4848 & $\mathrm{O}$ & 1 \\
\hline GB24 & 320 & .1593 & .3665 & $\mathrm{O}$ & 1 \\
\hline GB25 & 320 & .9187 & .2736 & $\mathrm{O}$ & 1 \\
\hline GB26 & 320 & .0562 & .2307 & $\mathrm{O}$ & 1 \\
\hline GB27 & 320 & .2500 & .4336 & 0 & 1 \\
\hline GB28 & 320 & .0468 & .2117 & o & 1 \\
\hline GB29 & 320 & .1625 & .3694 & O & 1 \\
\hline GB30 & 320 & .0812 & .2736 & O & 1 \\
\hline GB31 & 320 & .3687 & .4832 & $\mathrm{O}$ & 1 \\
\hline GB32 & 320 & .3156 & .4654 & O & 1 \\
\hline GB33 & 320 & .0593 & .2366 & $\mathrm{O}$ & 1 \\
\hline GB34 & 320 & .0593 & .2366 & $\mathrm{O}$ & 1 \\
\hline GB35 & 320 & .0062 & .0789 & O & 1 \\
\hline GB36 & 320 & .0031 & .0559 & $\mathrm{O}$ & 1 \\
\hline GB37 & 320 & .1250 & $\cdot 3312$ & $\mathrm{O}$ & 1 \\
\hline GB38 & 320 & .9500 & .2182 & $\mathrm{O}$ & 1 \\
\hline
\end{tabular}


Review of Economics and Development Studies, Vol. 7 (4) 2021, 543 - 559

All disclosure related items are binary in nature ranging from o-1 value. Among all 38 items 6 items are having maximum mean values ranging from .46 to .95. Some items are having very low level of average disclosure like .006-.002.

Table: 5 Correlation Matrix

According to correlation matrix it is clear that all variables in econometric model are perfectly uncorrelated with each other.

\begin{tabular}{|c|c|c|c|c|c|c|c|c|c|c|}
\hline & $\begin{array}{l}\text { Board } \\
\text { Size }\end{array}$ & $\begin{array}{l}\text { Board } \\
\text { Ind }\end{array}$ & $\begin{array}{l}\text { Female } \\
\text { Directo } \\
r\end{array}$ & IO & $\begin{array}{l}\text { Firm } \\
\text { Age }\end{array}$ & Lev & $R O A$ & $\begin{array}{l}\text { Firm } \\
\text { Size }\end{array}$ & $G B$ & $\begin{array}{l}\text { Tobin' } \\
s Q\end{array}$ \\
\hline $\begin{array}{l}\text { Board } \\
\text { Size }\end{array}$ & $\begin{array}{l}1.000 \\
0\end{array}$ & & & & & & & & & \\
\hline $\begin{array}{l}\text { Board } \\
\text { Ind }\end{array}$ & $\begin{array}{l}0.086 \\
6\end{array}$ & 1.0000 & & & & & & & & \\
\hline $\begin{array}{l}\text { Female } \\
\text { Directo } \\
\mathrm{r}\end{array}$ & $\begin{array}{l}0.195 \\
8\end{array}$ & 0.3632 & 1.0000 & & & & & & & \\
\hline $\mathrm{IO}$ & $\begin{array}{l}0.076 \\
9\end{array}$ & 0.6555 & 0.5204 & $\begin{array}{l}1.000 \\
0\end{array}$ & & & & & & \\
\hline $\begin{array}{l}\text { Firm } \\
\text { Age }\end{array}$ & $\begin{array}{l}0.007 \\
9\end{array}$ & 0.1794 & 0.0357 & $\begin{array}{l}0.114 \\
8\end{array}$ & $\begin{array}{l}1.000 \\
0\end{array}$ & & & & & \\
\hline Lev & $\begin{array}{l}0.230 \\
5\end{array}$ & 0.2514 & 0.2802 & $\begin{array}{l}0.263 \\
8\end{array}$ & $\begin{array}{l}0.285 \\
8\end{array}$ & $\begin{array}{l}1.000 \\
0\end{array}$ & & & & \\
\hline ROA & $\begin{array}{l}0.083 \\
8\end{array}$ & 0.2480 & 0.0555 & $\begin{array}{l}0.138 \\
7\end{array}$ & $\begin{array}{l}0.312 \\
6\end{array}$ & 0.1811 & $\begin{array}{l}1.000 \\
0\end{array}$ & & & \\
\hline $\begin{array}{l}\text { Firm } \\
\text { Size }\end{array}$ & $\begin{array}{l}0.043 \\
1\end{array}$ & 0.0379 & 0.1069 & .1121 & $\begin{array}{l}0.734 \\
1\end{array}$ & $\begin{array}{l}0.093 \\
4\end{array}$ & $\begin{array}{l}0.186 \\
6\end{array}$ & 1.0000 & & \\
\hline GB & $\begin{array}{l}0.150 \\
8\end{array}$ & 0.2608 & 0.0694 & .1037 & $\begin{array}{l}0.162 \\
5\end{array}$ & $\begin{array}{l}0.085 \\
5\end{array}$ & $\begin{array}{l}0.193 \\
5\end{array}$ & 0.0631 & $\begin{array}{l}1.000 \\
0\end{array}$ & \\
\hline $\begin{array}{l}\text { Tobin' } \\
\text { s Q }\end{array}$ & $\begin{array}{l}0.176 \\
0\end{array}$ & 0.0371 & 0.0323 & $\begin{array}{l}0.098 \\
6\end{array}$ & $\begin{array}{l}0.167 \\
0\end{array}$ & $\begin{array}{l}0.067 \\
5\end{array}$ & $\begin{array}{l}0.196 \\
6\end{array}$ & 0.2665 & $\begin{array}{l}0.007 \\
1\end{array}$ & $\begin{array}{l}1.000 \\
0\end{array}$ \\
\hline
\end{tabular}

A correlation among variable that exceeds 0.9 or VIF value greater than 10 shall indicate multi co-linearity (Gujarati, 2003). Table mentioned above shows there is no such issue among variables at all. 
Review of Economics and Development Studies, Vol. 7 (4) 2021, 543 - 559

Table: 6 Multi-Collinearity Diagnostic

\begin{tabular}{|l|l|l|}
\hline Variable & VIF & $\mathbf{1}$ VIF \\
\hline Firm Age years & 2.88 & 0.347719 \\
\hline Firm Size & 2.57 & 0.389607 \\
\hline IO & 2.17 & 0.459984 \\
\hline BoardInd & 1.97 & 0.507800 \\
\hline Female Director & 1.55 & 0.643527 \\
\hline Lev & 1.30 & 0.766859 \\
\hline GB & 1.23 & 0.811943 \\
\hline ROA & 1.22 & 0.822999 \\
\hline Board Size & 1.13 & 0.886665 \\
\hline Mean VIF & 1.78 & \\
\hline
\end{tabular}

\section{Empirical Result}

Principal component analysis is dimension reduction technique which is widely discussed in sustainability and CSR disclosure literature. (Benjamin et al, 2019). In this study, PCA provides 7 components with Eigenvalues $>1$. First component caries maximum information having eigenvalue 13.9 and explains 36.5\% variation which is very high. Rest of the 6 components collectively explains $34 \%$ variation. Rotated Matrix, eigenvectors and Scree plot of eigenvalues is also provided below. After identifying components predicted value of green banking index is calculated.

Table 7: Principal Component Analysis

\begin{tabular}{|l|l|l|l|l|}
\hline Component & Eigenvalue & Difference & Proportion & Cumulative \\
\hline Comp1 & 14.5792 & 10.9948 & .3738 & .3738 \\
\hline Comp2 & 3.58439 & 1.10395 & .0919 & .4657 \\
\hline Comp3 & 2.48044 & .0577924 & .0636 & .5293 \\
\hline Comp4 & 2.42265 & .664594 & .0621 & .5915 \\
\hline Comp5 & 1.75806 & .175874 & .0451 & .6365 \\
\hline Comp6 & 1.58218 & .352977 & .0406 & .6771 \\
\hline Comp7 & 1.22921 & .143176 & .0315 & .7086 \\
\hline
\end{tabular}


Figure-1: Scree Plot of eigenvalues after PCA

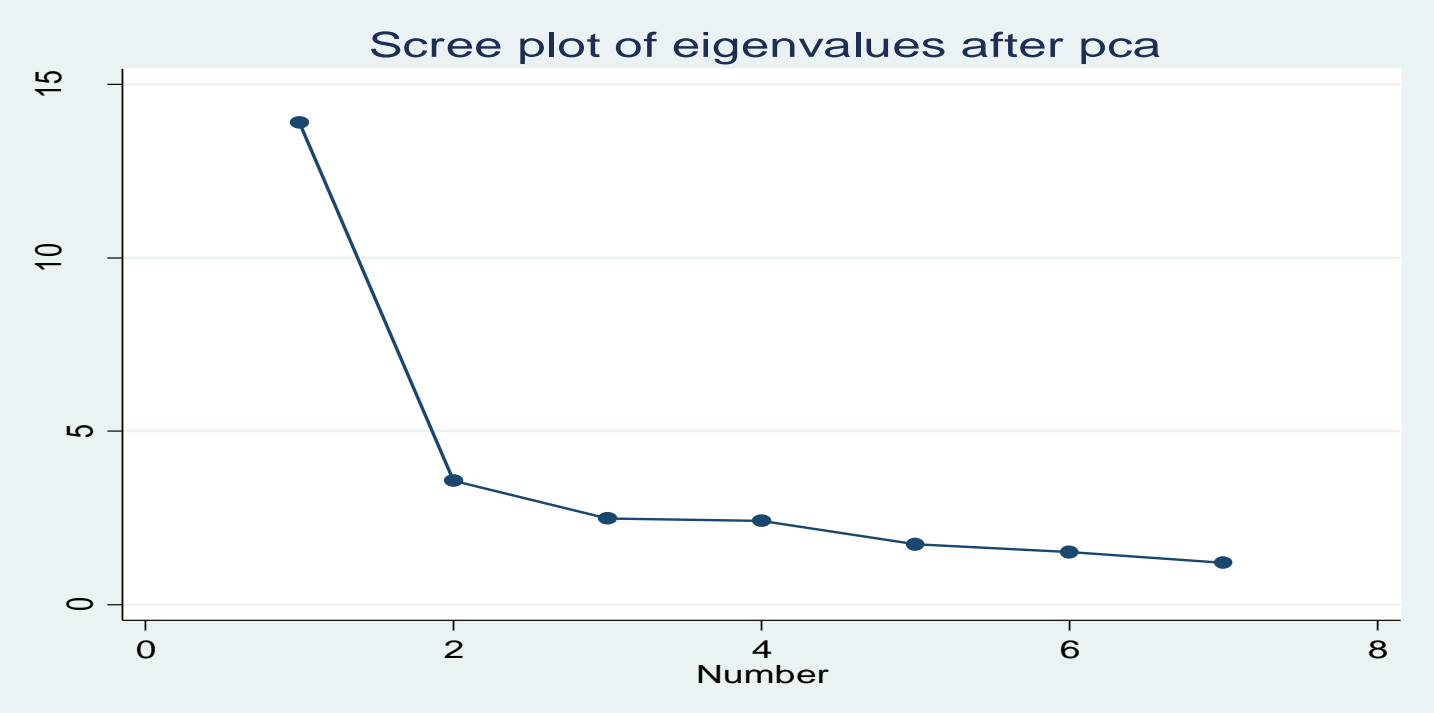

The scree plot is graphical representation of eigenvalues. The horizontal axis presents components and vertical axis presents eigenvalues while (Klomp and Haan, 2009). Figure-1 presents the eigenvalues of all three components and it can be observed that component-1 has the maximum value and produce the steep slope.

Table: 8 Principal Component Eigenvectors

\begin{tabular}{|l|l|l|l|l|l|l|l|}
\hline Variable & Comp1 & Comp2 & Comp3 & Comp4 & Comp5 & Comp6 & Comp7 \\
\hline GB1 & 0.2222 & -0.0647 & 0.0011 & -0.0304 & -0.0304 & -0.2008 & -0.1154 \\
\hline GB2 & 0.2237 & -0.0603 & -0.0292 & -0.0989 & -0.0989 & -0.0775 & -0.1712 \\
\hline GB3 & 0.2357 & -0.0629 & 0.0262 & -0.0470 & -0.0470 & -0.1836 & -0.0047 \\
\hline GB4 & 0.2199 & -0.1497 & -0.0385 & 0.0818 & 0.0818 & -0.0130 & -0.0186 \\
\hline GB5 & 0.2025 & 0.0380 & -0.0769 & -0.1206 & -0.1206 & 0.2378 & -0.0931 \\
\hline GB6 & 0.2089 & -0.1374 & -0.0109 & 0.0729 & 0.0729 & -0.0260 & 0.0711 \\
\hline GB7 & 0.1153 & -0.0732 & -0.0165 & 0.0176 & 0.0176 & 0.5210 & 0.0614 \\
\hline GB8 & 0.2248 & -0.0186 & -0.0489 & -0.0026 & -0.0026 & 0.0474 & 0.0688 \\
\hline GB9 & 0.2002 & -0.1023 & -0.0194 & 0.0907 & 0.0907 & -0.0208 & -0.3748 \\
\hline GB10 & 0.1886 & -0.1831 & -0.0258 & 0.0436 & 0.0436 & 0.1521 & -0.2347 \\
\hline GB11 & 0.1803 & 0.0175 & 0.0058 & -0.0632 & -0.0632 & -0.0359 & 0.1950 \\
\hline GB12 & 0.2180 & -0.0641 & 0.0266 & -0.0604 & -0.0604 & -0.1747 & 0.0790 \\
\hline GB13 & 0.2322 & -0.0544 & 0.0078 & -0.0465 & -0.0465 & -0.1166 & 0.0807 \\
\hline GB14 & 0.2092 & 0.0770 & -0.0141 & -0.0227 & -0.0227 & 0.0138 & 0.1849 \\
\hline GB15 & -0.1136 & 0.1789 & 0.1110 & 0.0671 & -0.0402 & 0.0180 & 0.0475 \\
\hline GB16 & -0.1136 & 0.1783 & 0.1110 & 0.0671 & -0.0402 & -0.1015 & 0.3950 \\
\hline GB17 & 0.1421 & -0.1777 & 0.2290 & 0.1376 & 0.1908 & -0.0536 & 0.0343 \\
\hline
\end{tabular}


Review of Economics and Development Studies, Vol. 7 (4) 2021, 543 - 559

\begin{tabular}{|l|l|l|l|l|l|l|l|}
\hline GB18 & 0.0588 & -0.0585 & -0.0890 & -0.0121 & -0.0216 & 0.0072 & -0.0731 \\
\hline GB19 & 0.0365 & 0.1775 & -0.0179 & 0.0459 & -0.0158 & 0.4550 & -0.2629 \\
\hline GB20 & 0.0362 & -0.0910 & 0.1961 & -0.1266 & -0.1661 & 0.0625 & -0.0300 \\
\hline GB21 & 0.1155 & 0.3341 & 0.0655 & -0.0107 & -0.0396 & -0.2370 & 0.0773 \\
\hline GB22 & 0.1891 & -0.0119 & 0.0544 & -0.3874 & 0.0990 & -0.0205 & -0.1834 \\
\hline GB23 & -0.1059 & -0.0330 & 0.1659 & -0.1020 & 0.2624 & 0.1260 & 0.1995 \\
\hline GB24 & 0.1848 & -0.1975 & 0.0005 & 0.0676 & -0.2862 & -0.0187 & 0.0330 \\
\hline GB25 & -0.0277 & -0.2335 & -0.0431 & -0.0568 & 0.0315 & -0.0221 & -0.0325 \\
\hline GB26 & -0.3763 & 0.0642 & 0.0041 & 0.4534 & -0.0370 & 0.1336 & 0.0256 \\
\hline GB27 & -0.0834 & 0.0168 & 0.1180 & 0.0288 & 0.1648 & -0.0301 & 0.0954 \\
\hline GB28 & 0.1155 & -0.0938 & 0.0934 & 0.0813 & 0.0041 & -0.0261 & 0.0244 \\
\hline GB29 & -0.0161 & 0.3069 & -0.0074 & 0.0296 & 0.0027 & 0.0417 & 0.0412 \\
\hline GB30 & -0.0292 & 0.0965 & -0.2042 & -0.0508 & -0.0077 & -0.0789 & -0.0714 \\
\hline GB31 & 0.2142 & -0.2149 & 0.0183 & 0.0142 & 0.1465 & 0.0273 & -0.1136 \\
\hline GB32 & 0.0059 & 0.0878 & -0.0422 & 0.2558 & -0.3320 & -0.2277 & -0.0377 \\
\hline GB33 & 0.0145 & -0.0573 & 0.1250 & 0.0159 & 0.0217 & 0.0588 & 0.0339 \\
\hline GB34 & 0.0145 & -0.0573 & 0.1250 & 0.0159 & 0.0217 & 0.0588 & 0.0339 \\
\hline GB35 & 0.0429 & -0.0914 & -0.0523 & -0.0181 & 0.0060 & -0.0127 & 0.0004 \\
\hline GB36 & -0.0587 & 0.1891 & -0.0224 & 0.0160 & 0.0060 & -0.0201 & -0.0446 \\
\hline GB37 & 0.1251 & -0.1471 & 0.0699 & -0.0266 & 0.0077 & 0.0871 & 0.0811 \\
\hline GB38 & 0.0806 & -0.0295 & 0.0012 & -0.0301 & -0.0064 & 0.0770 & -0.0251 \\
\hline
\end{tabular}

Table: 9 Principal Components Orthogonal Varimax Rotation

\begin{tabular}{|c|c|c|c|c|}
\hline \multicolumn{5}{|c|}{ No. of Obs: 320} \\
\hline \multicolumn{5}{|c|}{ No. of Comp:37 } \\
\hline \multicolumn{5}{|l|}{ Traces: 38} \\
\hline \multicolumn{5}{|l|}{ Rho:1.0000 } \\
\hline Component & Variance & Difference & Proportion & Cumulative \\
\hline Comp1 & 2 & 1 & 0.0526 & .0526 \\
\hline Comp2 & 1 & $1.02700 e-09$ & 0.0263 & .0780 \\
\hline Comp3 & 1 & $1.60119 \mathrm{e}-09$ & 0.0263 & .1053 \\
\hline Comp4 & 1 & $2.16254 \mathrm{e}-08$ & 0.0263 & .1316 \\
\hline Comp5 & 1 & $2.14184 \mathrm{e}-\mathrm{o} 8$ & 0.0263 & .1579 \\
\hline Comp6 & 1 & $2.78397 \mathrm{e}-11$ & 0.0263 & .1842 \\
\hline Comp7 & 1 & $9 \cdot 40026 \mathrm{e}-12$ & 0.0263 & .2105 \\
\hline Comp8 & 1 & $-7.27087 \mathrm{e}-11$ & 0.0263 & .2368 \\
\hline Comp9 & 1 & $7.04863 \mathrm{e}-11$ & 0.0263 & .2632 \\
\hline Comp10 & 1 & $3.07442 \mathrm{e}-09$ & 0.0263 & .2854 \\
\hline Comp11 & 1 & $-2.39225 \mathrm{e}-09$ & 0.0263 & .3158 \\
\hline Comp12 & 1 & $-6.80650 e-10$ & 0.0263 & .3421 \\
\hline Comp13 & 1 & $1.87759 \mathrm{e}-11$ & 0.0263 & .3684 \\
\hline
\end{tabular}


Review of Economics and Development Studies, Vol. 7 (4) 2021, 543 - 559

\begin{tabular}{|l|l|l|l|l|}
\hline Comp14 & 1 & $1.58955 \mathrm{e}-11$ & 0.0263 & .3947 \\
\hline Comp15 & 1 & $-9.25814 \mathrm{e}-09$ & 0.0263 & .4211 \\
\hline Comp16 & 1 & $8.80252 \mathrm{e}-09$ & 0.0263 & .4474 \\
\hline Comp17 & 1 & $4.08009 \mathrm{e}-10$ & 0.0263 & .4737 \\
\hline Comp18 & 1 & $1.53375 \mathrm{e}-10$ & 0.0263 & .5000 \\
\hline Comp19 & 1 & $-1.00031 \mathrm{e}-09$ & 0.0263 & .5263 \\
\hline Comp20 & 1 & $8.55693 \mathrm{e}-10$ & 0.0263 & .5789 \\
\hline Comp21 & 1 & $5.25580 \mathrm{e}-13$ & 0.0263 & .6053 \\
\hline Comp22 & 1 & $-2.17382 \mathrm{e}-13$ & 0.0263 & .6316 \\
\hline Comp23 & 1 & $5.51597 \mathrm{e}-11$ & 0.0263 & .6579 \\
\hline Comp24 & 1 & $2.13773 \mathrm{e}-08$ & 0.0263 &. .6842 \\
\hline Comp25 & 1 & $-2.14076 \mathrm{e}-08$ & 0.0263 & .7105 \\
\hline Comp26 & 1 & $-1.68218 \mathrm{e}-09$ & 0.0263 & .7368 \\
\hline Comp27 & 1 & $1.00998 \mathrm{e}-08$ & 0.0263 & .7632 \\
\hline Comp28 & 1 & $-2.88725 \mathrm{e}-09$ & 0.0263 & .7895 \\
\hline Comp29 & 1 & $-9.07525 \mathrm{e}-09$ & 0.0263 & .8158 \\
\hline Comp30 & $3.77244 \mathrm{e}-09$ & 0.0263 & .8421 \\
\hline Comp31 & 1 & $-7.68353 \mathrm{e}-09$ & 0.0263 & .8421 \\
\hline Comp32 & 1 & $7.41877 \mathrm{e}-09$ & 0.0263 & .8684 \\
\hline Comp33 & 1 & $-5.82249 \mathrm{e}-10$ & 0.0263 & .8947 \\
\hline Comp34 & 1 & $1.82356 \mathrm{e}-09$ & 0.0263 & .9211 \\
\hline Comp35 & 1 & $-1.55918 \mathrm{e}-09$ & 0.0263 & .97737 \\
\hline Comp36 & 1 & & 0.0263 & 1.000 \\
\hline Comp37 & 1 & & \\
\hline
\end{tabular}

Table 10: System GMM One-Step results for selected SAARC Countries: Corporate Governance characteristic and Market Value

\begin{tabular}{|c|c|c|c|c|c|c|}
\hline Tobin's Q & Coef. & Std. Err. & $\mathbf{z}$ & $\mathbf{P}>|\mathbf{z}|$ & \multicolumn{2}{|c|}{ [95\% Conf. Interval] } \\
\hline Tobin's Q L1 & .0528571 & .0472455 & 1.12 & .263 & -.0397424 & .1454566 \\
\hline Board Size & -6.335266 & 1.812093 & -3.50 & .000 & -9.886904 & -2.783628 \\
\hline Board Ind & 1.03113 & 3.280271 & 0.31 & .753 & $-5 \cdot 398083$ & $7 \cdot 460344$ \\
\hline Female Director & 3.285065 & 4.882335 & 0.67 & .501 & -6.284135 & 12.85427 \\
\hline IO & -.8228694 & .3143179 & -2.62 & .009 & -1.438921 & -.2068176 \\
\hline Firm Age & -2.004165 & .9467143 & -2.12 & .034 & -3.859691 & -.148639 \\
\hline Firm Size & 8.013257 & 2.072315 & -3.87 & .000 & 3.951594 & 12.07492 \\
\hline LEV & .5350474 & .4963865 & 1.87 & .281 & -.4378523 & -1.507947 \\
\hline ROA & -1.847115 & 3.636309 & 0.611 & .611 & -8.97415 & 5.279921 \\
\hline Obs. & \multicolumn{6}{|l|}{288} \\
\hline Wald Chi & \multicolumn{6}{|l|}{262.71} \\
\hline Prob & \multicolumn{6}{|l|}{0.000} \\
\hline Sargan test & \multicolumn{6}{|l|}{0.000} \\
\hline
\end{tabular}


Review of Economics and Development Studies, Vol. 7 (4) 2021, 543 - 559

Table 11: System GMM One-Step results for selected SAARC Countries: Green Banking, Corporate Governance Characteristics and Market Value.

\begin{tabular}{|c|c|c|c|c|c|c|}
\hline Tobin's $Q$ & Coef. & Std. Err. & $z$ & $P>|z|$ & \multicolumn{2}{|c|}{ [95\% Conf. Interval] } \\
\hline Tobin's Q L1 & .052121 & .0472671 & 1.10 & 0.270 & -.0405208 & .1447628 \\
\hline Board Size & -6.467463 & 1.81805 & -3.56 & 0.000 & -10.03078 & -2.90415 \\
\hline Board Ind & 1.515256 & $3 \cdot 322343$ & 0.46 & 0.648 & -4.996416 & 8.026928 \\
\hline Female Director & 3.016476 & 4.890703 & 0.62 & 0.537 & -6.569126 & 12.60008 \\
\hline $\mathrm{IO}$ & -.7357883 & .3276762 & -2.25 & -0.025 & -1.3780022 & -0.935548 \\
\hline Firm Age & -1.938855 & .9493205 & -2.04 & 0.041 & -3.799489 & -0.0782208 \\
\hline Firm Size & 8.023921 & 2.072781 & 3.87 & 0.000 & $3 \cdot 3961344$ & 12.0865 \\
\hline $\mathrm{LEV}$ & .5312251 & .4965618 & 1.07 & 0.285 & -.4420182 & 1.504468 \\
\hline ROA & -1.750552 & 3.638579 & -0.48 & 0.630 & -8.882037 & $5 \cdot 380952$ \\
\hline GB & -4.867581 & 5.214606 & -0.93 & 0.351 & -15.0880 & $15 \cdot 352859$ \\
\hline Obs. & \multicolumn{6}{|l|}{288} \\
\hline Wald Chi & \multicolumn{6}{|c|}{263.41} \\
\hline Prob & \multicolumn{6}{|l|}{0.000} \\
\hline Sargan test & \multicolumn{6}{|l|}{0.000} \\
\hline
\end{tabular}

To examine the relationship between board characteristics, green banking disclosure and firm value in selected SAARC countries STATA 14.2 software is used t. System GMM-step one method for panel data set covering the period 2010-2019 is applied. It has been recommended by Faitouri (2014) that one lag is sufficient to capture the influence of the past on the current data. First Data set is declared to be dynamic, panel ID is set to be banks and time is years. By clicking (ArellanoBover/Blundell-Bond estimation option, following command xtdpdsys generates results provided in table 5,6.After controlling the effects of firm specific characteristic such as, firm age, size, leverage and profitability at $5 \%$ confidence of interval board size has significant negative influence on market value of firm. $(\beta=6.33 p=.000)$. Institutional ownership has significant negative influence on market value. Green banking disclosure does not have any significant influence on market value. Lipton and Lorsch (1992) report that larger board size is ineffective. Agency theory (Jensen, 1993) suggests that large board size is dysfunctional. Optimum board size should be 8 or 7 . Beyond this limit board management is costly. The possible reason behind negative impact of board size is average size of board is 11-12 in SAARC countries collectively which is too high according to agency theory. (Jensen, 1993). Charfeddine and Elmarzougui (2010) identify negative impact of institutional ownership and firm performance measured as Tobin's Q in listed companies in France. According to controlling hypothesis institutional ownership beyond $81 \%$ contributes positively in firm value. (Wardhana and Tendililine, 2011).In present study average institutional ownership is between $21-22 \%$ which is very low. The conclusive findings are robust in the context of SAARC region.

\section{Conclusion}

The main contribution of this study is to shed light and explore dynamic relationships among green banking disclosure practices, corporate governance mechanism and firm value in selected SAARC Countries. Based upon author's knowledge, this is the first study which methodologically contributes by applying system GMM step one and PCA in the field of green banking disclosure. Contextually SARRC countries are targeted to explore unobserved dynamic relations as per research model. SAARC region is one of the most effected and threaded area due to climate risk and global warming. By developing a composite green banking disclosure index, a new stream in the field of disclosure is added. This index 


\section{Review of Economics and Development Studies, Vol. 7 (4) 2021, 543 - 559}

can be used as independent, mediator or moderator variable to explore unobserved relations with firm performance like market value, going concern value, profitability etc. Effectiveness of central bank's green banking guidelines can be observed in the light of theory of change and financial intermediation theory at regional and global level. Corporate governance mechanism and market value of firm is observed in the light of agency theory and controlling hypothesis. The findings are suggestive that corporate governance mechanism restructuring is needed to have positive contribution in market value of banks belonging to India, Pakistan, Bangladesh, Nepal and Sri Lanka.

\section{References}

Alqahtani, F. and Mayes, D.G. (2018). "Financial stability of Islamic banking and the global financial Crisis: evidence from the Gulf cooperation council”, Economic Systems, Vol. 42 No. 2, pp. 346-36o.

Al-Homaidi et al (2021). "Corporate Social Responsibility disclosure and profitability, Evidence from Islamic banks working in Yemen” Business properties Vol.2 PP, 91-102

Alexander K (2016.) Greening banking policy. In: Support of the G2o Green Finance Study Group Baltagi, B. (2008). Econometric Analysis of Panel Data, John Wiley and Sons.

Dahir, A.M., Mahat, F.B. and Ali, N.A.B. (2018). "Funding liquidity risk and bank risk-taking in BRICS countries", International Journal of Emerging Markets, Vol. 13 No. 1

Dewi \& Dewi (2017). “Corporate Social Responsibility, Green Banking, and Going Concern on Banking Company in Indonesia Stock Exchange”International Journal of Social Sciences and Humanities Vol. 1 No. 3, pp: 118-134

Faitouri (2014).“ Board of Directors and Tobin's Q: Evidence From U.K Firms” Journal of Finance And Accounting, Vol.2 No.4

Gosh, R. et al (2021). "Board Characteristics, audit committee attributes and firm performance: empirical evidence from emerging economy” Asian Journal of Accounting Research, Vol. 20 No.15

Gerged, A.M., Agwili, A. (2019),“ How Corporate Governance Affect Firm Value and Profitability?

Evidence from Saudi Financial and non-Financial listed firms” International Journal of Business Governance and Ethics, Vol. 14 No.2

Gujarati, D.N. (2003). Basic Econometrics, 4th ed., McGraw-Hill, New York, NY.

Islam S. et al (2017)."What Drives Green Banking Disclosure? An Institutional and Corporate Governance Perspective” Asia Pacific Journal of Management No.35, pp.: 501-527

Jatmiko et al (2020). "Impact of Corporate Governance Mechanism on Corporate Social Responsibility Disclosure of Publicly-Listed Banks in Bangladesh” Journal of Asian Finance Economics and Business Vol.7 No.6

Judson, R.A, Owen A,L. (1999).“ Estimating Dynamic Panel Data Models: A Guide for Macroeconomists” Economic Letters Vol.9 No.15

Karim et al (2020). “The Effects of Green Banking Practices on Financial Performance of Listed Banking Companies in Bangladesh" Canadian Journal of Business and Information studies, Vol2 No.6

Karyani E. and Obrien V.V(2020), “ Green Banking and Performance: The Role of Foreign and Public Ownership” Journal Dinamika Akuntansi dan Bisnis Vol.7 No.2

Klomp, J. and DE Haan, J. (2015), "Bank Regulation and Financial Fragility in Developing Countries Does Bank Structure Matter?”, Review of Development Finance, Vol. 5 No. 2, pp. 82-90.

Lipton, M. and Lorsch, J.W.(1992), “A Modest Proposal for Improved Corporate Governance” Business Lawyer,Vol.48. 
Review of Economics and Development Studies, Vol. 7 (4) 2021, 543 - 559

Monem et al (2021), "Does Green Banking Performance Pay Off? Evidence From A Unique Regulatory Setting in Bangladesh" Corporate Governance: An International Review No.29 No.2 pp162-187

Park H. \& Kim J.D (2020), “Transition Towards Green Banking: Role of Financial Regulators And Financial Institutions” Asian Journal of Sustainability and Social Responsibility Vol 5. No.5. Popa et al (2021), "Disclosure Dynamics and Non-Financial Reporting Analysis. The Case of Romanian Listed Companies" Sustainability Vol.13. No. 4732

Quazi et al (2021), "Green Banking Disclosure, Firm Value and Moderating Role a Contextual factor: Evidence from a Distinctive Regulatory Setting" Business Strategy and Environment.

Rehman, A. (2016), Al Baraka Bank Acquires Burj Bank, ProPakistani.

Osisioma and Nwokesi (2019), "Sustainability Disclosure and Market Value of Firms in Emerging Economy: Evidence from Nigeria” European Journal of Accounting, Auditing and Finance Research Vol.7 No.3

Sudhalakshmi K. \&Chinnadorai K.M (2014). "Green Banking Practices in Indian Banks"International Journal of Management and Commerce InnovationsVol. 2. No.1, pp: 232-235

Shaumya \& Arulrajah (2016). "Measuring Green Banking Practices: Evidence from Sri Lanka” 13th International Conference on Business Management.

Wu et al (2019). "Comparing the Influence of Green Credit on Commercial Bank Profitability in China and Abroad: Empirical Test Based on a Dynamic Panel System Using GMM" International journal of financial studies Vol.7 No.64

Appendix: 1 List of Items with key words developed by combining central Bank's guidelines.

\begin{tabular}{|l|l|}
\hline$\#$ & Item with Key words \\
\hline 1 & Board council promotes green credit. \\
\hline 2 & Low carbon business innovation. \\
\hline 3 & Bank own Environment \& Sustainable performance. \\
\hline 4 & Client supply chain impact on environment. \\
\hline 5 & Green Credit growth strategy. \\
\hline 6 & E\&S risk control. \\
\hline 7 & Information about Green Credit target. \\
\hline 8 & Green Credit follow-up report. \\
\hline 9 & Information about sector specific investment. \\
\hline 10 & Green Credit innovation. \\
\hline 11 & Stakeholder communication for awareness. \\
\hline 12 & Bank Own Environment \& sustainability improvement. \\
\hline 13 & Green offices promotion. \\
\hline 14 & Green Credit capacity building. \\
\hline 15 & $3^{\text {rd }}$ party Environment and Sustainability risk audit assessment. \\
\hline 16 & Client credit approval based on E\&S risk. \\
\hline 17 & Information regarding Internal audit based on green credit performance. \\
\hline 18 & Green credit incentive and penalty system. \\
\hline 19 & Green credit policy implementation status. \\
\hline 20 & Information about optional 3 ${ }^{\text {rd }}$ party independent audit. \\
\hline 21 & Email communication. \\
\hline
\end{tabular}


Review of Economics and Development Studies, Vol. 7 (4) 2021, 543 - 559

\begin{tabular}{|l|l|}
\hline 22 & Waste reduction policies including Water, gas etc. \\
\hline 23 & Energy consumption in conducting business operation. \\
\hline 24 & Employee travel reduction. \\
\hline 25 & Online, automated, mobile banking. \\
\hline 26 & Bank's network about environmental issues. \\
\hline 28 & Seminar and trainings about green banking. \\
\hline 29 & Bank award winning about environmental friendly activities. \\
\hline 30 & Internal marketing caption in annual report about green banking. \\
\hline 31 & Actual spending on green banking activities. \\
\hline 32 & Separate pages in annual report for green banking reporting. \\
\hline 33 & Green branch officer presence in bank branches. \\
\hline 34 & Green credit advisory services. \\
\hline 35 & Green credit financing targets at regional branch level. \\
\hline 36 & International funding for green project investments. \\
\hline 37 & Inventory targets for electricity, water, petroleum, paper. \\
\hline 38 & Paperless banking. \\
\hline
\end{tabular}

